\title{
Adjuvant tyrosine kinase inhibitors for renal cell carcinoma? No, thank you (at least for the present) reply
}

\author{
Hampig R Kourie*,1, Ziad Bakouny ${ }^{1}$, Fady GH Haddad ${ }^{1}$, Roland Eid ${ }^{1}$ \& Joseph Kattan ${ }^{1}$ \\ ${ }^{1}$ Hematology-Oncology Department, Faculty of Medicine, Saint Joseph University, Beirut, Lebanon \\ *Author for correspondence: hampig.kourie@usj.edu.lb
}

First draft submitted: 7 June 2018; Accepted for publication: 29 June 2018; Published online: 1 August 2018

Keywords: adjuvant $\bullet$ high-risk $\bullet$ pazopanib $\bullet$ renal cell carcinoma $\bullet$ tyrosine kinase $\bullet$ tyrosine kinase inhibitors

Dear Editor,

The authors would like to, first of all, thank our colleagues, the writers of the letter in response to our original meta-analysis [1] for their interest and insightful comments on the controversial role of tyrosine kinase inhibitors (TKIs) in the adjuvant treatment of renal cell carcinoma (RCC). Although the authors of the letter highlight the significant result of the fixed-effects model (HR: 0.91, 95\% CI: 0.83-0.99; $\mathrm{p}=0.03$ ), of the meta-analysis, readers should be reminded that the fixed-effects model was considered to be a sensitivity analysis in the original meta-analysis and that the random-effects model, which yielded a nonstatistically significant result (HR: 0.85, 95\% CI: $0.72-1.01 ; \mathrm{p}=0.06$ ), was considered to be the primary method of analysis. Although the two methods of analysis differed in that one was found to be statistically significant but not the other, there were no marked differences in the estimated hazard ratios (HR) but the results differed in the width of the confidence intervals, as would be expected in most cases where both analyses are undertaken [2]. As for the remaining comments, these can be answered by developments and data that were not available at the time of writing of the original meta-analysis.

First, the results of our meta-analysis differed from those of the meta-analysis by Gyawali et al. [3] and these differences were considered to be solely due to the addition of the results of the PROTECT trial [4]. While the authors of the letter accurately point out that the PROTECT trial failed to meet its primary end point of disease-free survival (DFS) in the pazopanib intention-to-treat (ITT) $600 \mathrm{mg}$ population, the fact that the results of the trial were not significant does not preclude the finding of an overall significant effect of TKIs on DFS due to increased power with the addition of these results to the meta-analysis [2]. Moreover, the results of the pazopanib $800 \mathrm{mg}$ ITT population of the PROTECT trial had also been included in the meta-analysis for completeness and since the two treatment arms had separate control groups (and therefore the inclusion of all four arms in the meta-analysis was possible without giving a disproportionate weight to one of the arms). Furthermore, although the 3-year DFS of the control arm of the ITT $800 \mathrm{mg}$ population was worse than that of the ITT $600 \mathrm{mg}$ population, there is no evidence that there was improper randomization between the arms of the ITT $800 \mathrm{mg}$ population (especially that the clinical and demographic characteristics of the two arms, reported in the PROTECT trial paper, seem to be comparable) [4].

Second, the authors of the letter highlight an important issue; the discordance between the definitions of highrisk RCC between the three trials included in the meta-analysis [4-6]. It should be underlined that all three papers specifically mentioned in the aims of their studies that they were investigating the effect of a TKI on the risk of relapse in patients with 'high-risk' RCC. However, the definition of what constitutes 'high-risk' RCC varied between the different studies and this was reflected by the varying inclusion criteria between the different studies. Furthermore, although the higher-risk subset of the ASSURE trial did not significantly benefit from either sunitinib or sorafenib, this lack of treatment effect should be considered with caution considering that the analysis was not adequately powered to detect an effect in this subgroup and that this analysis was not preplanned and therefore the compared groups may have not been balanced [7]. These issues have been discussed in greater depth in a recent review article by Figlin et al. [8]. 
Third, the authors recognize that the results of the sponsored trials may have possibly been influenced by conflicts of interest relating to sponsorship. However, the authors do not believe that this factor should be considered as the sole determinant of the observed between-study discrepancies, especially since a number of other differences between the studies may have accounted for these discrepancies. In particular, the higher cumulative dosing in the S-TRAC study (with no mid-study drug dose reduction, contrarily to the two other trials), blinded central review at both baseline and recurrence only in the S-TRAC study, and higher-risk RCC in the S-TRAC study compared with both the PROTECT and ASSURE studies, as well as the inclusion of only clear cell RCC in both the PROTECT and S-TRAC studies versus the ASSURE study which also included nonclear cell RCC $[4-6,8]$ may partially account for the observed between-study differences. Moreover, the discrepancy between the independent central review results and those of the investigators in the S-TRAC study would have probably called the results of the study more into question had the differences between the two methods of review been larger and especially if only the investigator review results had been significant [6].

Fourth, a major drawback for the use of TKIs in the adjuvant treatment of RCC is that none of them has been shown to increase overall survival (OS). DFS is often used as a surrogate for OS in clinical trials in the adjuvant setting and formed the basis for the approval of the use of sunitinib by the US FDA in the adjuvant treatment of high-risk RCC. However, a recent trial-level meta-analysis found only a modest correlation between 5-year DFS and 5-year OS with an $\mathrm{R}^{2}$ of 0.48 (95\% CI: 0.14-0.67), which is below the usual threshold of surrogacy of $\mathrm{R}^{2}$ $\geq 0.7$ [9]. OS may also be heavily influenced by postprotocol therapies that may be unbalanced between groups and some advocate for DFS as a more direct reflection of treatment effect, although this remains controversial $[8,10]$. Furthermore, in the case of the three adjuvant TKI trials, OS data were often immature at the time of analysis [4-6].

Fifth, the authors recognize that TKIs are not currently a standard of care for the adjuvant treatment of high-risk RCC and based on the results of the meta-analysis, they would not be able to recommend TKIs for all patients with resected high-risk RCC. However, considering the lack of currently available options, the authors would suggest discussion about the potential risks and benefits of adjuvant TKIs with patients who are able-bodied, who understand and are willing to risk adverse events, who are at higher risk for recurrence, and who understand the relatively small benefit that may be procured by TKIs. Finally, it should be noted that the controversial nature of this topic is best illustrated by the approval of sunitinib in the adjuvant treatment of high-risk RCC by the FDA and its refusal by the European Medical Agency.

In conclusion, the authors would not advocate for the use of TKIs systematically in the adjuvant treatment of all high-risk RCC patients; however, in the absence of currently available alternatives in this setting, the findings of our recently published meta-analysis suggest that TKIs could remain an option for well-selected patients. A patient-level meta-analysis could precisely determine which patient should be selected and whether the benefit of TKIs in this setting is significant.

Financial \& competing interests disclosure

The authors have no relevant affiliations or financial involvement with any organization or entity with a financial interest in or financial conflict with the subject matter or materials discussed in the manuscript. This includes employment, consultancies, honoraria, stock ownership or options, expert testimony, grants or patents received or pending, or royalties.

No writing assistance was utilized in the production of this manuscript.

\section{References}

1 Kourie HR, Bakouny Z, Eid R, Haddad FG, Kattan J. The merit of tyrosine kinase inhibitors in the adjuvant setting of high-risk renal cell carcinoma: a meta-analysis. Future Oncol. 14(9), 829-835 (2018).

2 Deeks J, Higgins J, Altman D. Chapter 9: analysing data and undertaking meta-analyses. In: Cochrane Handbook for Systematic Reviews of Interventions Version 5.1.0. Higgins JPT, Green S (Eds). The Cochrane Collaboration, London, UK (2011).

3 Gyawali B, Ando Y. Adjuvant sunitinib for high-risk resected renal cell carcinoma: a meta-analysis of ASSURE and S-TRAC trials. Ann. Oncol. 28(4) 898-899 (2017)

4 Motzer RJ, Haas NB, Donskov F, Gross-Goupil M, Varlamov S. Randomized Phase III trial of adjuvant pazopanib versus placebo after nephrectomy in patients with locally advanced renal cell carcinoma (RCC) (PROTECT). J. Clin. Oncol. 35(Suppl.), Abstract 4507 (2017).

5 Haas NB, Manola J, Uzzo RG et al. Adjuvant sunitinib or sorafenib for high-risk, non-metastatic renal-cell carcinoma (ECOG-ACRIN E2805): a double-blind, placebo-controlled, randomised, Phase III trial. Lancet 387(10032), 2008-2016 (2016). 
6 Ravaud A, Motzer RJ, Pandha HS et al. Adjuvant sunitinib in high-risk renal-cell carcinoma after nephrectomy - supplementary index. N. Engl. J. Med. 375(23), 2246-2254 (2016).

7 Haas NB, Manola J, Dutcher JP et al. Adjuvant treatment for high-risk clear cell renal cancer updated results of a high-risk subset of the ASSURE randomized trial. 19104(9), 1249-1252 (2018).

8 Figlin RA, Leibovich BC, Stewart GD, Negrier S. Adjuvant therapy in renal cell carcinoma: does higher risk for recurrence improve the chance for success? 29(2) 324-331 (2018).

9 Harshman LC, Xie W, Moreira RB et al. Evaluation of disease-free survival as an intermediate metric of overall survival in patients with localized renal cell carcinoma: a trial-level meta-analysis. Cancer 124(5), 925-933 (2017).

10 Kemp R, Prasad V. Surrogate endpoints in oncology: when are they acceptable for regulatory and clinical decisions, and are they currently overused? BMC Med. 15(1), 134 (2017). 
